\title{
O PROBLEMA DE ROTEAMENTO NO TRANSPORTE ESCOLAR
}

\author{
* Maria Teresinha Arns Steiner \\ **Luzia Vidal S. Zamboni, \\ ***Deise M. Bertholdi Costa \\ *Celso Carnieri \\ *Arinei Lindbeck da Silva \\ Universidade Federal do Paraná \\ Departamentos : * Matemática e **Desenho \\ Caixa Postal 19002 - CEP 81531-990 \\ Curitiba - Paraná - Brasil
}

\section{Resumo}

O presente trabalho aborda o problema de roteamento no transporte escolar e descreve algumas técnicas da Pesquisa Operacional que podem ser utilizadas para solucioná-lo. O problema considera além das distâncias a serem percorridas por $m$ veículos, a disponibilidade e capacidades destes e, além disso, as demandas em cada um dos $n$ pontos de demanda. A implementação a um problema real é estudada e os resultados analisados.

Palavras-chave: Problema de roteamento de veículos, clusters, rotas ótimos.

\begin{abstract}
This paper deals with the vehicle routing problem for school children transportation and describes some Operations Research techniques that can be used to solve it. The problem considers, besides the distances to be traveled by $m$ vehicles, the vehicle availabilities and capacities and the demands in each of the $n$ points. The implementation to a real problem is studied and the results are analised.
\end{abstract}

Keywords: Vehicle Routing Problem, clusters, optimal routes. 


\section{INTRODUÇÃO}

O problema de roteamento clássico de veículos necessita de um conjunto de rotas de coleta e/ou entrega a partir de um depósito central para vários pontos de demanda, cada um tendo necessidades de serviços, com o objetivo de minimizar a distância total a ser percorrida pela frota inteira. Os veículos têm restrições de quantidade, capacidade e, possivelmente, de tempo. Todos os veículos iniciam e terminam sua rota em um depósito central. A formulação deste problema como um Problema de Programação Inteira Binária (PPIB) é devida a Golden et al., 1977 [Bodin et al., 1983]. Como o problema de roteamento de veículos é NP-hard, resolvê-lo como um PPIB é inviável e, por este motivo, a solução é obtida, em geral, através de procedimentos heurísticos.

O tratamento dado aos problemas de roteamento de veículos é bastante diversificado, variando não apenas nos algoritmos utilizados, mas também no tipo de tratamento dado às particularidades próprias de cada problema. Bodin et al., 1983, fazem uma descrição do problema do roteamento no transporte escolar para escolas americanas, onde um mesmo ônibus pode atender várias escolas, sendo que cada escola tem um conjunto de pontos de demanda, com localizações próximas às residências dos alunos, com um dado número de alunos designados a cada ponto. Bowerman et al., 1995, também abordam o problema de roteamento de ônibus escolar urbano. Graciolli, 1994, faz a abordagem do problema de roteamento na coleta de resíduos sólidos de serviços de saúde. Renz, 1994, aborda o problema de roteamento com restrições de tempos de viagens e de trabalho para equipes de inventário florestal, responsáveis por coleta sistemática de informações. E assim poderse-ia citar muitos outros trabalhos de roteamento de veículos, pois este é um dos assuntos mais estudados na área de Pesquisa Operacional [Bodin et al., 1983].

O problema de roteamento no transporte escolar em foco neste trabalho, descrito na seção 2 , foi tratado através da junção de vários procedimentos heurísticos da Pesquisa Operacional, conforme apresentados na seção 3, onde são evidenciados os detalhes necessários para considerar as particularidades do problema de forma a obter-se boa performance para os referidos procedimentos. Na seção 4 é feita a implementação dos procedimentos heurísticos ao problema real e os resultados são analisados. Finalmente, na seção 5, são apresentadas as conclusões e sugestões para trabalhos futuros.

\section{DESCRIÇÃO DO PROBLEMA REAL}

Algumas escolas particulares na cidade de Curitiba, PR, possuem uma frota de veículos composta por ônibus, micro-ônibus e/ou vans, que pode ser própria ou terceirizada, para prestar atendimento aos seus alunos quanto ao serviço de coleta e/ou entrega dos alunos nas suas residências (casas, apartamentos, condomínios e outros).

A escola pesquisada possui 29 ônibus próprios de diversas capacidades, variando de 32 até 60 lugares, que atendem a 997 alunos na data da pesquisa. Destes 29 ônibus, a escola utiliza apenas 24, sendo que os demais permanecem como reserva para situações emergenciais. São em número de 12 os tipos de ônibus (conforme a capacidade), de acordo com o Quadro 1, na seção 4. Estes veículos partem da garagem aproximadamente uma hora antes do início das aulas, apanham um certo número de alunos e se dirigem à escola. Como a escola pesquisada se situa na região metropolitana de Curitiba, os ônibus permanecem na escola após a coleta, retornando para a cidade apenas no final das aulas para o serviço de entrega dos alunos. 
São 997 alunos concentrados em 717 pontos de demanda, ou seja, muitos dos pontos de demanda possuem mais de um usuário. Há alunos que são irmãos, bem como alunos que moram em um mesmo edifício. A máxima demanda constatada em um ponto de demanda foi de 10 alunos.

A escola faz a localização de todos os alunos em um mapa da cidade. Sobre o mapa, o gerente de transportes da escola, com o auxílio de um taxista conhecedor das particularidades da cidade e dos sentidos das vias, define os roteiros, de forma a atender todos os alunos. A solução adotada pela escola encontra-se no Quadro 1, onde se vê, por exemplo, que a escola utiliza 2 dos 3 ônibus do tipo 1, com capacidade para 32 lugares, sendo que as demandas são de 27 e 30 alunos cada um. As distâncias percorridas para este ônibus são, respectivamente, $10.523 \mathrm{~m}$ e $17.953 \mathrm{~m}$.

Conforme mencionado, o objetivo do presente trabalho é fornecer à escola uma solução otimizada, que minimize, além da distância total percorrida pela frota de ônibus, o número destes, considerando as suas capacidades e as particularidades do problema.

Para isto, o trabalho foi dividido em 5 fases :

1a. Fase: Localização das residências dos alunos em um mapa digitalizado da cidade de Curitiba obtendo-se, desta forma, as coordenadas geográficas para cada um dos pontos de demanda. Na Figura 1 tem-se os pontos de demanda e também a localização da garagem dos ônibus e da escola;

2a. Fase: Obtenção da quantidade e respectivas capacidades ótimas dos veículos necessários para atender a demanda.

3a. Fase: Determinação dos clusters ótimos de atendimento, ou seja, definição de quais pontos de demanda serão atendidos por cada um dos veículos da frota. Numa $1^{\text {a }}$ etapa, sem considerar as capacidades dos ônibus e em uma $2^{\text {a }}$ etapa, fazendo esta consideração;

4a. Fase: Obtenção dos roteiros em cada cluster de atendimento, ou seja, obtenção da sequência em que os pontos de demanda devem ser atendidos;

5a. Fase: Aplicação de melhorias nos roteiros obtidos na 4a. Fase, evitando-se cruzamentos entre rotas e cruzamentos dentro de uma rota;

Os resultados para as fases de 2 a 5 foram obtidos através da implementação das heurísticas apresentadas na seção 3 .

\section{HEURÍSTICAS ABORDADAS PARA A SOLUÇÃO DO PROBLEMA REAL}

Nesta seção são apresentadas as heurísticas utilizadas neste trabalho com o objetivo de solucionar o problema de roteamento no transporte escolar pesquisado. Na seção 3.1 é apresentado um modelo de um Problema de Programação Linear Inteira (PPLI) para a obtenção da quantidade necessária de ônibus de cada tipo a serem utilizados pela escola para atender a demanda. Na seção 3.2 é apresentado um procedimento para a obtenção de sementes (que farão o "papel" de depósitos artificiais) com o objetivo de inicializar a formação dos clusters de atendimento, ou seja, dos agrupamentos de pontos de demanda que serão atendidos pelos ônibus. Nas seções 3.3 e 3.4 estão descritos procedimentos para a obtenção destes clusters ótimos; primeiramente sem considerar as restrições de capacidade dos veículos (1- $1^{\text {a. }}$ etapa), e depois, considerando as capacidades ( $2^{\text {a. }}$ etapa). 
O objetivo na formação dos clusters é concentrar os pontos de demanda, evitando-se assim que um aluno fique muito tempo no veículo. Com uma melhor concentração dos pontos de demanda, o tempo que cada ônibus leva desde a sua saída da garagem até o primeiro ponto de demanda de seu cluster não é contabilizado para o aluno. Além disso, todos os alunos de um mesmo cluster terão aproximadamente o mesmo tempo de permanência dentro do ônibus.

Definidos os clusters de atendimento, na seção 3.5 é descrito o procedimento de construção de rota utilizado neste trabalho, o algoritmo da inserção mais econômica. Para fazer uso deste algoritmo, foi considerado como primeiro nó de cada rota, o ponto de demanda mais próximo da garagem e como último nó de dada rota, o ponto de demanda mais próximo da escola, sendo que os demais pontos de demanda foram inseridos entre o inicial e final pelo referido algoritmo de inserção. E, na seção 3.6, são apresentados procedimentos de melhorias para se evitar cruzamentos entre e dentro das rotas.

Em cada uma das seguintes sub-seções, de 3.1 a 3.6, são apresentados os algoritmos heurísticos abordados e, na mesma sub-seção, a forma como os referidos algoritmos foram utilizados para resolver o problema estudado.

\subsection{O PPLI para a obtenção da Quantidade Ótima de Ônibus de cada Tipo}

Para inicializar a solução do problema, obteve-se a quantidade e capacidades ótimas dos ônibus a serem utilizados através de um PPLI considerando a disponibilidade de todos os veículos (quantidades e capacidades) e a demanda total de alunos.

O objetivo deste modelo, apresentado a seguir, é minimizar a quantidade de ônibus, e consequentemente, estar-se-á minimizando o número de motoristas e os custos operacionais com a frota, considerando que a frota de veículos é própria e que os veículos encontram-se em igual estado de conservação.

$$
\operatorname{Min}_{x} \mathrm{z}=\sum_{i=1}^{\text {ntipos }} \mathrm{x}_{i}
$$

sujeito a :

$$
\begin{aligned}
& \sum_{i=1}^{\text {ntipos }} \operatorname{Cap}_{i} \quad \mathrm{x}_{i} \geq \mathrm{Q} \\
& \mathrm{x}_{i} \leq \mathrm{q}_{i} \quad(i=1, \ldots, \text { ntipos }) \\
& \mathrm{x}_{i} \geq 0 \text { e inteiro }
\end{aligned}
$$

onde as variáveis de decisão $\mathrm{x}_{i}$ são as quantidades de ônibus de cada tipo disponíveis pela escola,

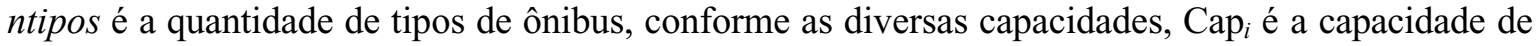
cada tipo de ônibus, Q é a quantidade total de alunos a serem atendidos e $\mathrm{q}_{i}$ é a quantidade de ônibus disponível de cada tipo.

Utilizando os dados do Quadro 1, o modelo do PPLI para o problema abordado fica estabelecido como se segue: 


$$
\underset{x}{\operatorname{Min}} \mathrm{z}=\sum_{i=1}^{12} \mathrm{x}_{i}
$$

sujeito a :

$$
\begin{aligned}
& \sum_{i=1}^{12}\left(\operatorname{Cap}_{i}-\mathrm{f}\right) \mathrm{x}_{i} \geq \mathrm{Q} \\
& \mathrm{x}_{i} \leq \mathrm{q}_{i} \quad(i=1, \ldots, 12) \\
& \mathrm{x}_{1} \geq 2 \\
& \mathrm{x}_{i} \geq 0 \text { e inteiro }
\end{aligned}
$$

onde a folga f é colocada com o objetivo de nas fases seguintes poder-se fazer um melhor ajuste na formação dos clusters. Esta folga $\mathrm{f}$ assume valores variando de 2 a 6 lugares, que corresponde de $5 \%$ a $15 \%$ da capacidade de um veículo médio (40 lugares). Das 4 alternativas testadas nos procedimentos subsequentes (obtenção das sementes e formação dos clusters ótimos), a alternativa com folga igual a 4 foi a que apresentou melhores resultados, pelos motivos expostos na seção 3.4.

A restrição (3.4-b) do modelo é devido a uma necessidade particular da escola: ter disponíveis dois micro-ônibus para o atendimento da região central da cidade, onde ônibus grandes não circulam ou o fazem com dificuldade.

A solução ótima para este modelo forneceu 24 ônibus, número este coincidente com o número já utilizado pela escola. Observe-se que a quantidade e capacidades dos veículos foram obtidas apenas para se inicializar o problema, já que depois, como será visto na seção 3.4, estes dados serão remanejados.

\subsection{Obtenção das Sementes (depósitos artificiais)}

Para inicializar a formação dos clusters, precisa-se fazer a determinação de $m$ sementes, uma para cada ônibus. Entre os diversos procedimentos existentes na literatura, adotou-se o apresentado por Paraíba, 1990:

Passo 1. Faça $\mathrm{k}=1$. Encontre $\mathrm{i}_{\mathrm{k}}$, tal que $\mathrm{c}\left(0, \mathrm{i}_{\mathrm{k}}\right)=\max \{\mathrm{c}(0, \mathrm{i})\}$, para todo $\mathrm{i}$, onde 0 é o depósito central, ou seja, encontre o ponto de demanda mais distante do depósito central;

Passo 2. Enquanto $1 \leq \mathrm{k}<\mathrm{m}$, faça:

$\mathrm{k}=\mathrm{k}+1$. Encontre o ponto de parada $\mathrm{i}^{*}$, ainda não selecionado tal que :

$\mathrm{c}\left(\mathrm{i}, \mathrm{i}_{\mathrm{k}-1}\right)+\mathrm{c}\left(\mathrm{i}, \mathrm{i}_{\mathrm{k}-2}\right)+\ldots+\mathrm{c}\left(\mathrm{i}, \mathrm{i}_{2}\right)+\mathrm{c}\left(\mathrm{i}, \mathrm{i}_{1}\right)$ seja máximo para $\mathrm{i}=\mathrm{i}^{*}$.

Faça $i_{k}=i^{*}$.

$\mathrm{O}$ algoritmo descrito determina as sementes de forma que estejam o mais longe uma das outras, evitando a concorrência na subsequente formação dos clusters.

Como o problema abordado não possui um depósito central, e além disso, necessita de clusters para os pontos de demanda localizados na região central da cidade, fez-se o seguinte:

- primeiramente foram definidas sementes para a região central da cidade. Os locais para estas sementes foram escolhidos manualmente, fazendo-se, desta forma, uma interação entre o tomador de decisões e o algoritmo. Cada uma destas sementes centrais agrupou os 30 alunos mais próximos, determinados pela distância euclidiana. Para estes 2 clusters iniciais ficou definida uma folga $f$ de 2 lugares em cada um, para eventuais ajustes subsequentes, conforme explanado na seção 3.4. 
- em seguida, pelo algoritmo apresentado anteriormente, foram determinadas as demais 22 sementes para o restante da cidade, onde foi considerado como depósito central, nó 0 , uma (das duas) sementes anteriormente escolhidas na região central da cidade e, assim, obtiveram-se as 24 sementes, uma para cada veículo. O que aconteceu, porém, foi que a partir da obtenção da $8^{a}$ semente, as suas localizações começaram a ficar muito próximas, ou seja, as sementes passaram a ser concorrentes para a aglutinação dos pontos de demanda. Optou-se, então, por ficar com as 7 primeiras sementes, com localizações bem diferenciadas. Na Figura 2 tem-se a localização das sementes, onde as sementes centrais são as sementes 1 e 2 , e as outras 7 , obtidas pelo algoritmo, foram numeradas de 3 a 9 .

\subsection{Procedimento para a obtenção dos clusters ótimos - 1a. Etapa}

Obtidas as sementes, aplicou-se o procedimento proposto por Gillett e Johnson, 1973 [Bodin et al., 1983], [Golden et al., 1977] para a obtenção dos clusters:

Inicialmente, todos os pontos encontram-se sem designação.

Para cada nó i, seja t’(i) a semente mais próxima a i, e t’(i) a segunda semente mais próxima a i.

Para cada nó i, a razão: $r(i)=c\left(i, t^{\prime}(i)\right) / c\left(i, t^{\prime \prime}(i)\right)$ é calculada e todos os nós são colocados em ordem crescente pelos valores de r(i), e assim fica determinada a ordem na qual os nós são designados a uma semente. Aqueles que estão relativamente próximos a uma semente são considerados primeiro. Depois que um certo número de nós foram designados da lista de r(i), um pequeno cluster é formado ao redor de cada semente.

Os nós i cuja razão r(i) está próxima de 1 , ou seja, i é um nó que pode ser agrupado por t' ou t”, são designados como se segue. Se dois nós adjacentes $\mathrm{j}$ e $\mathrm{k}$ já estão designados a uma semente, inserindo $i$ entre $\mathrm{j}$ e $\mathrm{k}$ na rota ligada a $\mathrm{t}(\mathrm{t}$ ' ou t'), cria um custo adicional igual a $\mathrm{c}(\mathrm{j}, \mathrm{i})+\mathrm{c}(\mathrm{i}, \mathrm{k})-\mathrm{c}(\mathrm{j}$, k) ao custo total. Ou seja, o algoritmo designa o nó i a uma semente $t$ ( $t$ ' ou t') inserindo i entre dois nós já designados a semente $t$ ( $t$ ' ou $\left.t^{\prime \prime}\right)$ de maneira que minimize os custos. O nó i será designado à semente $t$ ( $t$ ' ou $\left.t^{\prime \prime}\right)$ que fornecer o menor custo adicional.

No problema abordado, fez-se a designação dos pontos de demanda às 7 sementes definidas em 3.2 seguindo o procedimento anteriormente proposto, não esquecendo que para as 2 sementes da região central da cidade, já tinham sido designados 30 alunos. Nesta fase não foram consideradas as capacidades dos veículos, mas simplesmente o cálculo da razão $r(i)$ como apresentado anteriormente para se fazer a designação. Para os pontos i duvidosos, com r(i) $>0.7$, fez-se a designação de acordo com a expressão $\mathrm{c}(\mathrm{j}, \mathrm{i})+\mathrm{c}(\mathrm{i}, \mathrm{k})-\mathrm{c}(\mathrm{j}, \mathrm{k})$. Desta forma, ficou-se com 2 clusters com demanda de 30 alunos e 7 grandes clusters cujas demandas ficaram superiores às capacidades dos ônibus.

A designação dos pontos de demanda nesta $1^{a}$ etapa tem por objetivo fornecer uma solução inicial para a aplicação do algoritmo apresentado na seção 3.4 a seguir, o qual estabelece a formação definitiva dos clusters.

\subsection{Procedimento para a obtenção dos clusters ótimos - 2a. Etapa}

Este procedimento, devido a Tillman e Cain, 1972 [Tillman e Cain, 1972], [Bodin et al., 1983], começa com uma solução inicial consistindo por servir cada nó exclusivamente por uma rota a partir do depósito mais próximo. 
A partir desta solução inicial, o procedimento consta basicamente do seguinte: seja c(i, j) o custo ou distância entre os nós i e j; c(i, k) o custo ou distância entre o nó i e o depósito k. O custo total inicial de todas as rotas é dado por

$$
\mathrm{D}=\sum_{i=1}^{n} 2 \underset{k}{\operatorname{Min}}\{\mathrm{c}(\mathrm{i}, \mathrm{k})\} .
$$

O método liga sucessivamente pares de nós sempre visando um custo total mínimo. Uma regra básica é assumida no algoritmo: a designação inicial dos nós ao depósito mais próximo é temporária, mas assim que dois ou mais nós tenham sido designados a uma rota comum a partir de um mesmo depósito, os nós não podem ser redesignados a outro depósito. Além disso, assim como no algoritmo original dos savings de Clarke e Wright, 1964, os nós i e j podem ser ligados somente se i e j não forem pontos interiores de uma rota existente.

A cada passo, a escolha de ligar um par de nós i e j em uma rota a partir de um depósito $\mathrm{k}$ é feita em termos dos savings. Os nós i e j podem ser ligados somente se as restrições de capacidade e de ligação não forem violadas. O cálculo dos savings não é tão simples quanto no caso de um único depósito. Os savings $\mathrm{s}(\mathrm{i}, \mathrm{j}, \mathrm{k})$ estão associados com todas as combinações dos nós i e j e depósito $\mathrm{k}$, e representa o decréscimo no custo total ou distância viajada quando se liga i e j a k. A fórmula é dada por :

$s(i, j, k)=c^{\prime}(i, k)+c^{\prime}(j, k)-c(i, j)$,

onde c'(i, k) $=2 \min _{t}\{\mathrm{c}(\mathrm{i}, \mathrm{t})\}-\mathrm{c}(\mathrm{i}, \mathrm{k})$, se i ainda não recebeu uma designação permanente; $\mathrm{c}(\mathrm{i}, \mathrm{k})$, caso contrário.

No primeiro caso, o nó i está sendo redesignado do seu depósito mais próximo e a designação previamente feita de custo $2 \min \{\mathrm{c}(\mathrm{i}, \mathrm{t})\}$ é economizada; no segundo caso, o nó j está sendo inserido entre o depósito $\mathrm{k}$ e o nó i e a ligação de i a k é retirada da solução atual.

Os savings $\mathrm{s}(\mathrm{i}, \mathrm{j}, \mathrm{k})$ são calculados para $\mathrm{i}, \mathrm{j}=1, \ldots, n(\mathrm{i} \neq \mathrm{j})$ e $\mathrm{k}=1, \ldots, \mathrm{m}$ a cada passo. Eles são armazenados em $m$ matrizes de ordem $n \times n$. A cada passo, a ligação $\mathrm{i}-\mathrm{j}$ em $\mathrm{k}$ é escolhida tal que maximize $s(i, j, k)$ e que produza uma solução viável (capacidade e outras restrições).

Seguindo o procedimento descrito para o problema abordado, tem-se já a solução inicial para o problema determinada na seção 3.3. Inicialmente tem-se $n$ rotas, sendo que estas rotas vão sendo ligadas de acordo com o cálculo dos savings anteriormente apresentado, fazendo o devido controle das capacidades dos ônibus. Ao se finalizar o processo de formação de rotas, alguns dos pontos de demanda não tinham se ligado a nenhuma das rotas formadas, e, ao invés de se ter a formação de 24 rotas, obtiveram-se 29 rotas, pois 5 pontos de demanda ficaram isolados, cada um em uma rota formada por apenas ele próprio e o depósito ao qual estava temporariamente ligado. Para contornar este problema, utilizaram-se as folgas previstas em cada uma das rotas. Os lugares nos veículos, correspondentes a estas folgas, passaram a ficar "liberados" a partir deste momento, ou seja, cada ônibus passou a usufruir de sua capacidade total.

Para fazer bom uso desta capacidade "adicional", foram designados os pontos isolados em ordem decrescente de demanda, ou seja, quanto maior a demanda, maior foi a prioridade para se fazer a designação, garantindo-se desta forma, melhores inserções para estes pontos isolados. Foi nesta etapa que verificou-se que para uma folga de 4 lugares obtinha-se, para este problema, uma 
melhor solução: além de menor distância total, obteve-se maior homogeneidade nas demandas dos ônibus, ou seja, os veículos ficaram com a taxa de ocupação semelhante.

Fez-se também, nesta etapa, o remanejamento dos ônibus, pois as vezes ocorria o fato de ônibus menores terem maior quantidade de alunos do que ônibus maiores, ou seja, alocaram-se as demandas dos clusters em ordem crescente aos ônibus com capacidades em ordem crescente também. O resultado deste procedimento, ou seja, a definição dos clusters finais, está na Figura 3.

Obtidos os clusters ótimos, deve-se obter o roteiro para cada veículo, ou seja, definir a sequência na qual a coleta de alunos deve ser feita, e para isto foi utilizado o algoritmo heurístico de Inserção mais Econômica, descrito a seguir.

\subsection{Algoritmo Heurístico para Construção de Rota - Inserção mais econômica}

O algoritmo heurístico da Inserção mais Econômica, devido a Rosenkrantz, Sterns e Lewis, 1977 [Golden et al., 1980], [Bodin et al., 1983], considera uma rota com k nós na iteração $\mathrm{k}$ e determina qual nó, que ainda não está na rota, poderia ser o próximo a ser ligado à rota (seleção) e então determina onde na rota ele deveria ser inserido (inserção). Para isso, são adotados os seguintes passos:

Passo 1. Comece com um sub-grafo consistindo somente do nó i;

Passo 2. Ache um nó k tal que c(i, k) seja mínimo e forme a sub-rota i-k-i;

Passo 3. Passo da Seleção e Inserção. Ache (i,j) na sub-rota e k que não esteja na rota, tal que $c(i, k)+c(k, j)-c(i, j)$ seja mínimo e, então, insira k entre i e j;

Passo 4. Se um circuito Hamiltoniano tiver sido formado, pare. Caso contrário, volte ao passo 3.

O algoritmo da inserção mais econômica foi aplicado separadamente para cada um dos clusters formados em 3.4, considerando sempre as distâncias euclidianas entre os pontos de demanda.

Esgotados os estágios dos algoritmos heurísticos, ou seja, construidos os roteiros para cada um dos ônibus, propõe-se um refinamento baseado em trocas de pontos entre rotas ou ainda, em trocas na sequência de pontos numa mesma rota de tal forma a obter uma redução do custo total, evitando cruzamentos entre rotas e dentro de uma mesma rota, respectivamente. Estes refinamentos estão descritos na seção 3.6 a seguir.

\subsection{Procedimentos para melhoria da solução}

Dos numerosos procedimentos existentes para melhoria de rotas, o presente trabalho faz a abordagem e a aplicação do procedimento de troca de pontos entre duas rotas, descrito por Paraíba, 1990, e do procedimento das melhorias 2-opt e 3-opt em uma rota, desenvolvido por Lin e Kernighan, 1973, apresentados a seguir:

Procedimento 1 : Troca de pontos entre duas rotas $R_{s}$ e $R_{k}$ :

Seja $\mathrm{s}_{\mathrm{t}} \in \mathrm{R}_{\mathrm{s}}$ (localizado entre $\mathrm{s}_{\mathrm{t}-1}$ e $\mathrm{s}_{\mathrm{t}+1}$ ) e $\mathrm{k}_{\mathrm{m}} \in \mathrm{R}_{\mathrm{k}}$ (localizado entre $\mathrm{k}_{\mathrm{m}-1}$ e $\mathrm{k}_{\mathrm{m}+1}$ ). Se 


$$
\mathrm{c}\left(\mathrm{s}_{\mathrm{t}-1}, \mathrm{k}_{\mathrm{m}}\right)+\mathrm{c}\left(\mathrm{k}_{\mathrm{m}}, \mathrm{s}_{\mathrm{t}+1}\right)+\mathrm{c}\left(\mathrm{k}_{\mathrm{m}-1}, \mathrm{~s}_{\mathrm{t}}\right)+\mathrm{c}\left(\mathrm{s}_{\mathrm{t}}, \mathrm{k}_{\mathrm{m}+1}\right)<\mathrm{c}\left(\mathrm{s}_{\mathrm{t}-1}, \mathrm{~s}_{\mathrm{t}}\right)+\mathrm{c}\left(\mathrm{s}_{\mathrm{t}}, \mathrm{s}_{\mathrm{t}+1}\right)+\mathrm{c}\left(\mathrm{k}_{\mathrm{m}-1}, \mathrm{k}_{\mathrm{m}}\right)+\mathrm{c}\left(\mathrm{k}_{\mathrm{m}}, \mathrm{k}_{\mathrm{m}+1}\right)
$$

então troque $s_{t}$ por $k_{m}$ na rota $R_{s}$ e $k_{m}$ por $s_{t}$ na rota $R_{k}$.

Se este procedimento for repetido para todos os pares de pontos, então ele reduz a interseção entre os roteiros.

Procedimento 2 : Troca de pontos em uma mesma rota:

Os procedimentos 2-opt e 3-opt para trocas em uma rota consistem no seguinte: para um dado k, define-se uma k-troca de uma rota consistindo da deleção de $\mathrm{k}$ arcos de uma rota que serão trocados por k outros arcos de modo a formar uma nova rota. Uma rota é k-ótima se não for mais possível efetuar trocas para melhorar a distância total viajada. Geralmente o 3-opt se aproxima bastante da solução ótima.

Os procedimentos 1 e 2 foram aplicados ao problema pesquisado até que não fosse mais possível efetuar trocas entre as rotas e nas rotas.

Os resultados das heurísticas adotadas, demandas de cada ônibus e distâncias euclidianas percorridas, encontram-se no Quadro 1. Para exemplificar, a Figura 4 mostra o roteiro obtido para um dos ônibus, a nível de quadra após o procedimento de inserção e de melhorias.

\section{IMPLEMENTAÇÃO DAS HEURÍSTICAS E ANÁLISE DOS RESULTADOS}

Para o problema real de roteamento no transporte escolar, apresentado na seção 2, utilizando-se as técnicas e as respectivas particularidades necessárias descritas na seção 3, implementadas em linguagem Visual Basic 4.0, obteve-se a solução apresentada no Quadro 1, nas colunas da Solução Otimizada. Para medir-se o grau de eficiência das técnicas abordadas, os resultados obtidos foram comparados com a solução realizada pela escola, atentando para o fato de que em ambas soluções foram consideradas as distâncias euclidianas.

No Quadro 1 verifica-se que tanto a solução da escola quanto a solução otimizada fez uso de 24 ônibus, observando que um dos ônibus do tipo 4, com capacidade para 43 lugares, faz 2 viagens, na solução da escola.

Vê-se, por exemplo no Quadro 1, que dos 3 ônibus do tipo 1, cuja capacidade é de 32 lugares, a escola utiliza 2 que percorrem $10.523 \mathrm{~m}$ e $17.953 \mathrm{~m}$, ocupando 27 e 30 lugares, respectivamente. Já a solução otimizada também faz uso de 2 destes ônibus ocupando 30 lugares cada um deles, sendo que cada um dos ônibus deverá percorrer $3.351 \mathrm{~m}$ e $6.537 \mathrm{~m}$, respectivamente. E assim temse a leitura dos demais resultados.

Através destes resultados observa-se que o percentual de melhoria ao adotar-se os procedimentos de otimização é bastante significativo, sendo de aproximadamente 25\% ((451.594 - 338.258) / 451.594). Esta melhoria foi obtida, principalmente, devido a maneira de se concentrar os pontos de demanda.

Na Figura 5, tem-se a visualização de todas as rotas para a solução otimizada. 
SOLUÇÃO DA ESCOLA SOLUÇÃO OTIMIZADA

\begin{tabular}{|c|c|c|c|c|c|c|}
\hline $\begin{array}{c}\text { Tipo do } \\
\text { Onibus } \\
(\mathrm{i})\end{array}$ & $\begin{array}{c}\text { Quantidade } \\
\left(\mathrm{q}_{\mathrm{i}}\right)\end{array}$ & $\begin{array}{c}\text { Capacidade } \\
\left(\mathrm{cap}_{\mathrm{i}}\right)\end{array}$ & \begin{tabular}{c} 
Demanda \\
\hline 1
\end{tabular} & $\begin{array}{c}\text { Distância percorrida } \\
(\mathrm{m})\end{array}$ & Demanda & $\begin{array}{c}\text { Distância percorrida } \\
(\mathrm{m})\end{array}$ \\
\hline 2 & 1 & 32 & $27 / 30$ & $\begin{array}{c}10.523 / \\
17.953\end{array}$ & $30 / 30$ & $3.351 / 6.537$ \\
\hline 3 & 2 & 41 & $41 / 32$ & $\begin{array}{c}28.343 / \\
18.008\end{array}$ & $30 / 34$ & $24.961 / 13.912$ \\
\hline 4 & 6 & 43 & $34 / 72 * / 43$ & $8.450 / 51.072 /$ & $37 / 40 / 36 /$ & $\begin{array}{c}8.669 / 13.476 / 14.895 / \\
10.761 / 13.364\end{array}$ \\
\hline 5 & 2 & 44 & 40 & 34.289 & $40 / 34$ & $18.799 / 23.735$ \\
\hline 6 & 1 & 45 & 45 & 28.107 & 42 & $13.880 / 17.167$ \\
\hline 7 & 1 & 47 & --- & --- & 44 & 24.831 \\
\hline 8 & 8 & 49 & $45 / 46 / 47 /$ & $9.874 / 20.355 /$ & $44 / 44 / 44 /$ & $15.000 / 10.396 / 6.748 /$ \\
& & & $41 / 42 / 44 /$ & $24.449 / 9.873 /$ & $44 / 44 / 44 /$ & $10.353 / 10.423 / 9.200 /$ \\
& & & $47 / 41$ & $25.560 / 12.719 /$ & 47 & 13.187 \\
\hline 9 & 2 & 50 & $48 / 48$ & $48.971 / 24.798$ & 48 & 24.560 \\
\hline 10 & 1 & 56 & 55 & 27.106 & 51 & 6.235 \\
\hline 11 & 1 & 58 & 55 & 8.337 & 53 & 5.452 \\
\hline 12 & 1 & 60 & 55 & 2.780 & 55 & 5.383 \\
\hline & & & & & & \\
\hline Total & 29 & 1315 & 997 & 451.594 & 997 & 338.258 \\
\hline
\end{tabular}

Quadro 1. Comparação entre a solução adotada pela escola e a solução otimizada

* Trata-se de um mesmo ônibus que faz 2 viagens.

\section{CONCLUSÕES E SUGESTÕES PARA FUTUROS TRABALHOS}

Dentre as diversas formas possíveis de se resolver o problema de roteamento no transporte escolar, foi adotada uma metodologia, neste trabalho, que consta basicamente das seguintes etapas:

- fez-se a localização dos pontos de demanda em um mapa digitalizado de maneira exata: não apenas considerando rua, bairro, mas também a quadra e o lado da quadra (direito ou esquerdo). Com isto, obtiveram-se as coordenadas geográficas de cada ponto de demanda;

- obtiveram-se, em seguida, as sementes que fazem o "papel" dos depósitos (fictícios) dos algoritmos abordados para a formação de clusters de atendimento de acordo com a quantidade de veículos;

- obtiveram-se os clusters para cada uma das sementes;

- formaram-se as rotas envolvendo os pontos de demanda de cada cluster;

- finalmente, fizeram-se melhorias nestas rotas.

As melhorias obtidas através da solução otimizada em relação a solução da escola foram muito significativas, como pode ser constatado no Quadro 1, e não podem ser desprezadas. Ao se minimizar a distância total percorrida pela frota de ônibus, obtém-se como consequências economia de combustível e de manutenção dos ônibus além da minimização do tempo de permanência dos alunos nos ônibus, fator considerado como sendo de maior importância para a escola e maior satisfação por parte dos pais e alunos. 
Deve-se considerar, entretanto, que uma boa solução matemática, apresentando elevado percentual de melhoria, pode não significar que seja uma boa solução para a escola, que possui muitas particularidades na sua forma de atendimento como, por exemplo, motoristas que estão acostumados com a sua região de atendimento e não aceitam grandes mudanças; ou então pais que exigem que seu filho seja atendido por determinado motorista, ou ainda, exigência de horários, em que os motoristas não podem seguir a sequência de pontos otimizada para entrega e/ou coleta.

Além deste problemas, pode-se dizer que a maior dificuldade encontrada para a implementação deste trabalho na prática foi, sem dúvida, o fato da solução precisar sofrer ajustes no decorrer do ano, devido a atualização frequente na localização dos pontos de demanda, ocorrendo de 2 a 3 atualizações semanais. Estas atualizações ocorrem pela inclusão, exclusão e, principalmente, devido a mudanças nos endereços dos alunos. A solução vai sendo modelada, de acordo com estas alterações, sempre tentando minimizar as alterações nos roteiros dos ônibus, normalmente obtendose soluções mais distantes da solução ótima, até que toda a solução deva ser remodelada, a cada semestre, por exemplo.

Como complemento a este trabalho, precisa-se ainda "transformar" os roteiros obtidos considerando apenas as distâncias euclidianas, em roteiros quadra a quadra, isto é, considerando os sentidos das vias, mãos e contra-mãos, obtendo-se assim, os roteiros reais e suas respectivas distâncias reais.

Seria, também, bastante interessante, comparar os procedimentos heurísticos utilizados com as outros desenvolvidos mais recentemente, como por exemplo, Algoritmos Genéticos [Goldberg, 1989], Redes Neurais [Hopfield, 1984] e Simulated Annealing [Kirkpatrick et al., 1983]. Por se tratar de procedimentos heurísticos, ter-se-á uma infinidade de soluções para o problema de roteamento no transporte escolar; quais e de que maneira estes procedimentos devem ser adotados de forma a obter uma boa solução deve ser uma preocupação constante no tratamento de todo problema de roteamento.

Os autores agradecem a Maxi Data Tecnologia em Informática pela disponibilização do mapa digitalizado da cidade de Curitiba, PR, para a realização deste trabalho.

\section{REFERÊNCIAS BIBLIOGRÁFICAS}

BALlOU, R. H. Logística Empresarial. São Paulo, Editora Atlas, 1992.

BODIN, L., GOLDEN, B., ASSAD, A. and BALL, M. Routing and Scheduling of vehicles and crews : the state of the art. England, Pergamon Press, vol. 10. n. 2, 1983 (Special Issue).

BOWERMAN, R., Hall, B. and CALAMAI, P. A Multi-Objective Optimization approach to Urban School Bus Routing: Formulation and Solution Method, Transportation Research, vol. 29, n. 2, p. 107-123, 1995.

CLARKE, G. and WRIGHT, J. W. Scheduling of Vehicles from a Central Depot to a Number of Delivery Points, Operations Research, vol. 12, n. 4, p. 568-581, 1964.

GOLDBERG, D. Genetic Algorithms in Search. Optimization and Machine Learning. Reading, Addison-Wesley, 1989. 
GOLDEN, B. L. , MAGNANTI, T. L. , NGUYEN, H. Q. Implementing vehicle routing algorithms, Networks, v. 7, p. 113-148, 1977.

GOLDEN, B. , BODIN, L. , DOYLE, T. , STEWART Jr, W. Approximate traveling salesman algorithms, Operations Research, v. 28, n. 3, parte II, p. 694-711, 1980.

GRACIOLLI, O. D. Planejamento de Rpteiros de veículos Coletores de Resíduos Sólidos de Serviços de Saúde. Florianópolis, Universidade Federal de Santa Catarina, 1994. (Dissertação de Mestrado - Engenharia de Produção).

HOPFIELD, J. J. Neurons with graded response have collective computational properties like those of two-state neurons. Proc. Natl. Acad. Sci. USA, 3088-3092, 1984.

KIRKPATRICK, S. , GELATT, Jr C. D. , VECCHI, M. P. Optimization by Simulated Annealing, Science, 220, p. 671-680, 1983.

LIN, S. and KERNIGHAN, B. W. An Effective Heuristic Algorithm for the Traveling Salesman Problem, Operations Research, v. 21, p. 498-516, 1973.

NOVAES, A. G. Sistemas Logísticos - Transporte, Armazenagem e Distribuição Física de Produtos. São Paulo, Edgard Bucler Ltda., 1989.

PARAÍBA, L. C., FERNANDES, J. F. R. e ANDO, A. S. Um Algoritmo Heurístico de Construção Paralela para o Problema do M-Caixeiro Viajante. Trabalho de circulação interna - Departamento de Engenharia Elétrica e Sistemas, UNICAMP, Campinas, 1990.

RENZ, L. C. Um Algoritmo para Roteirização com Restrições de Tempos de Viagens e de Trabalho. Florianópolis, Universidade Federal de Santa Catarina, 1994. (Dissertação de Mestrado Engenharia de Produção).

STEINER, M. T. A., COSTA, D. M. B., ZAMBONI, L. V. S., LINDBECK da SILVA, A. e CARNIERI, C. The Vehicle Routing Problem in the School Transportation, Anais do Congresso, EURO XV / INFORMS XXXIV, Barcelona, Espanha, 1997.

TILLMAN, F. A. , CAIN, T. M. An upperbound algorithm for the single and multiple terminal delivery problem, Management Science, v. 18, n. 11, p. 664-682, 1972.

ZAMBONI, L. V. S. Técnicas de Roteirização de Veículos aplicadas ao Transporte Escolar. Curitiba, Universidade Federal do Paraná, 1997. (Dissertação de Mestrado - Métodos Numéricos em Engenharia / Programação Matemática). 


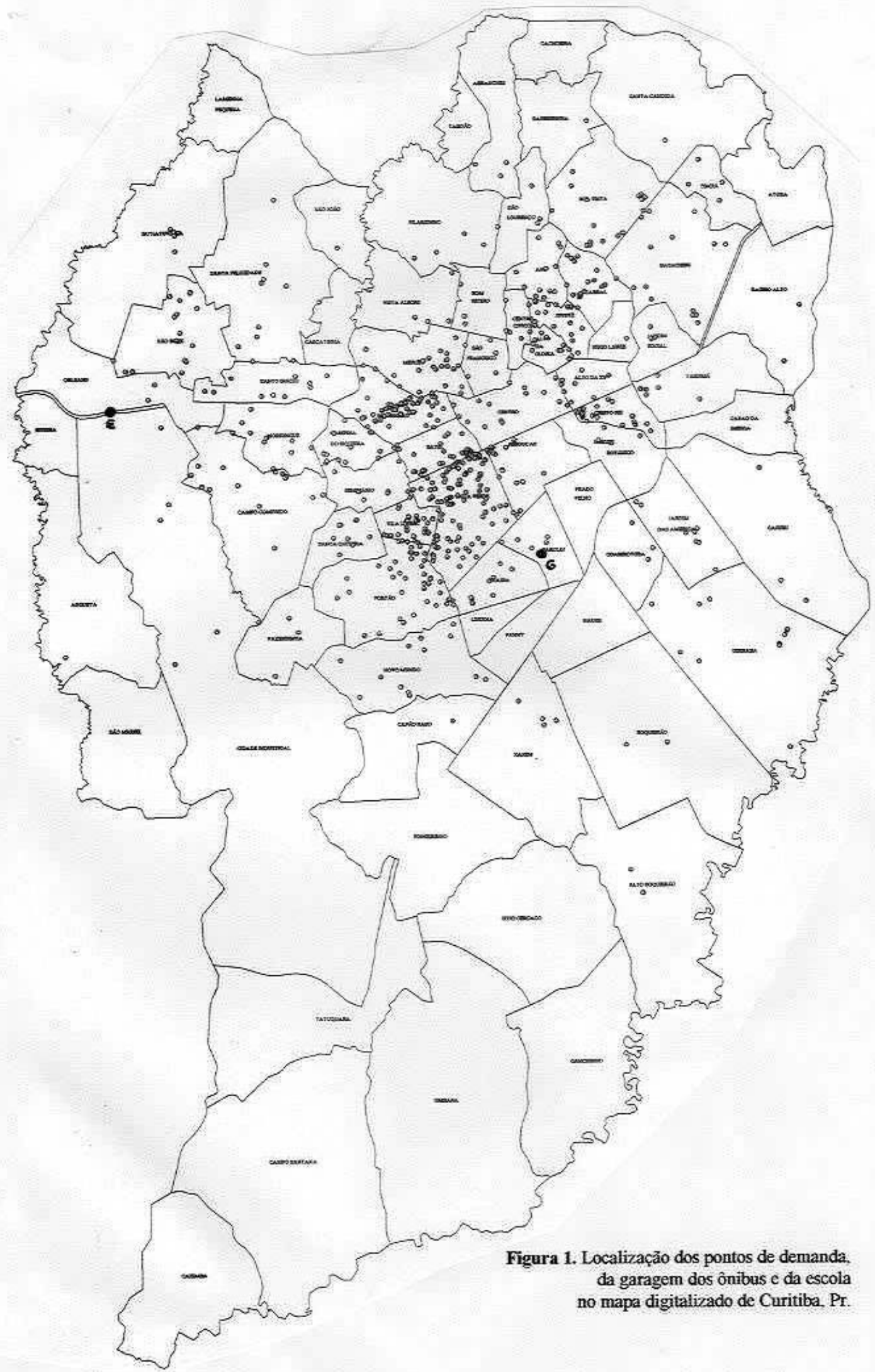




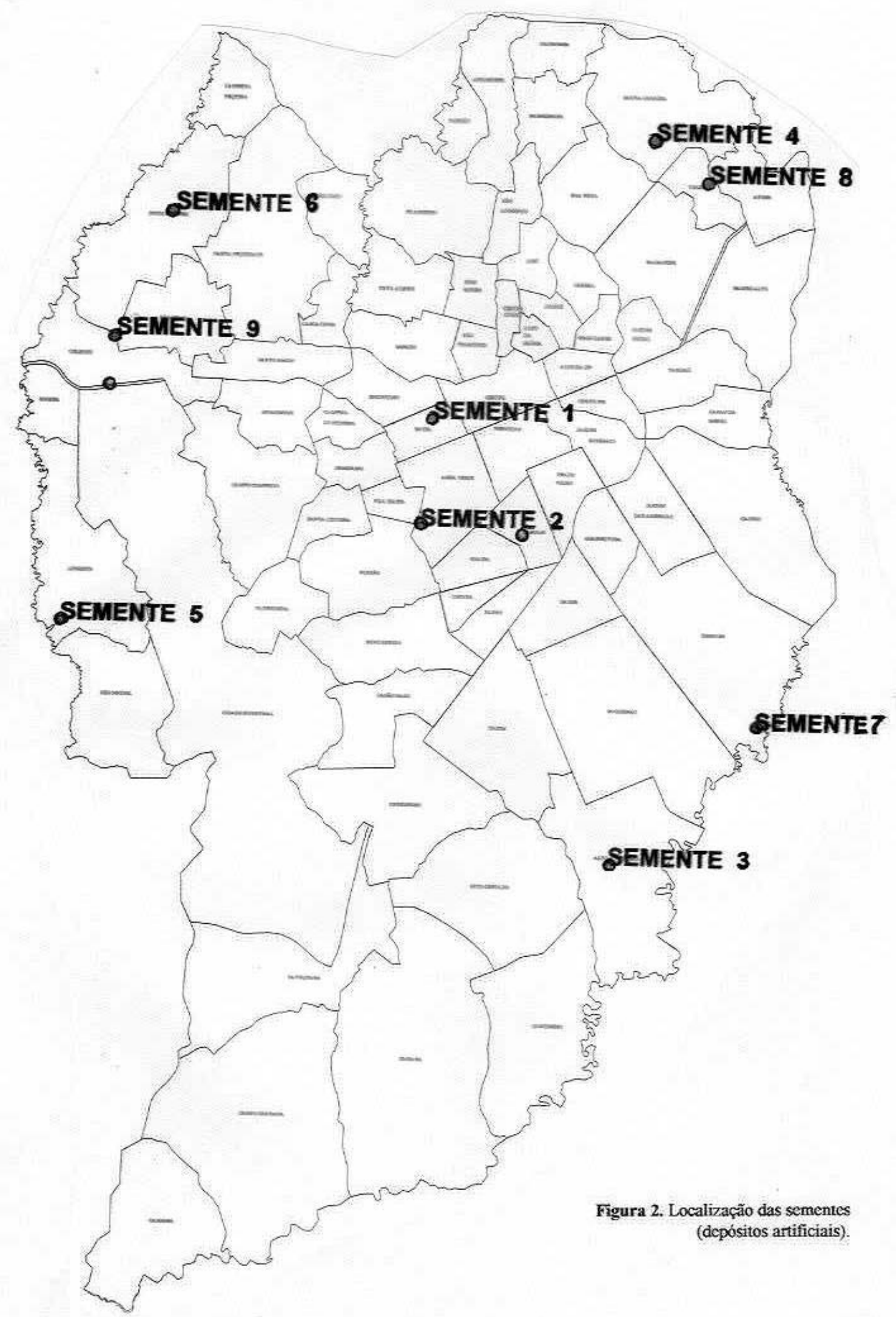




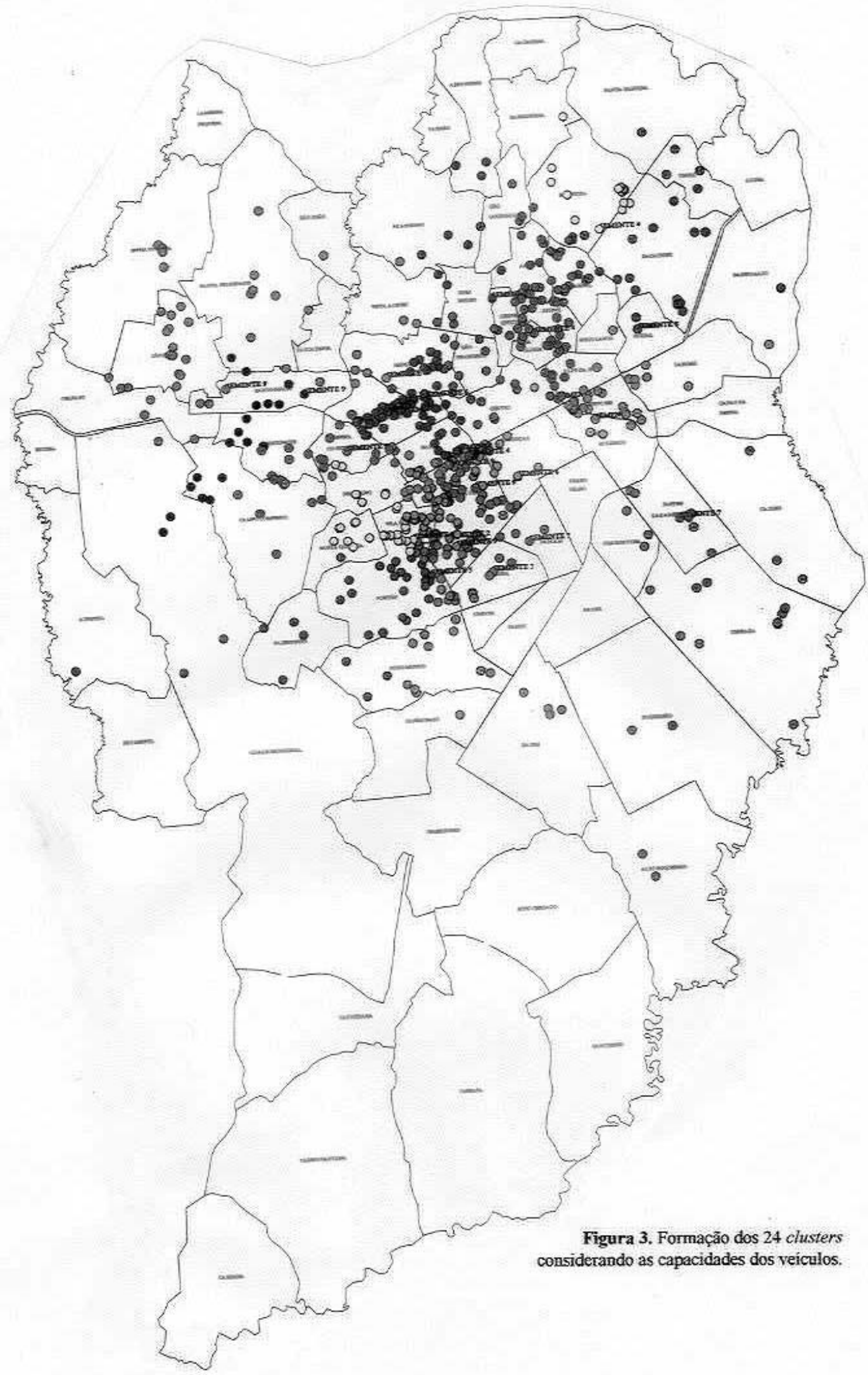




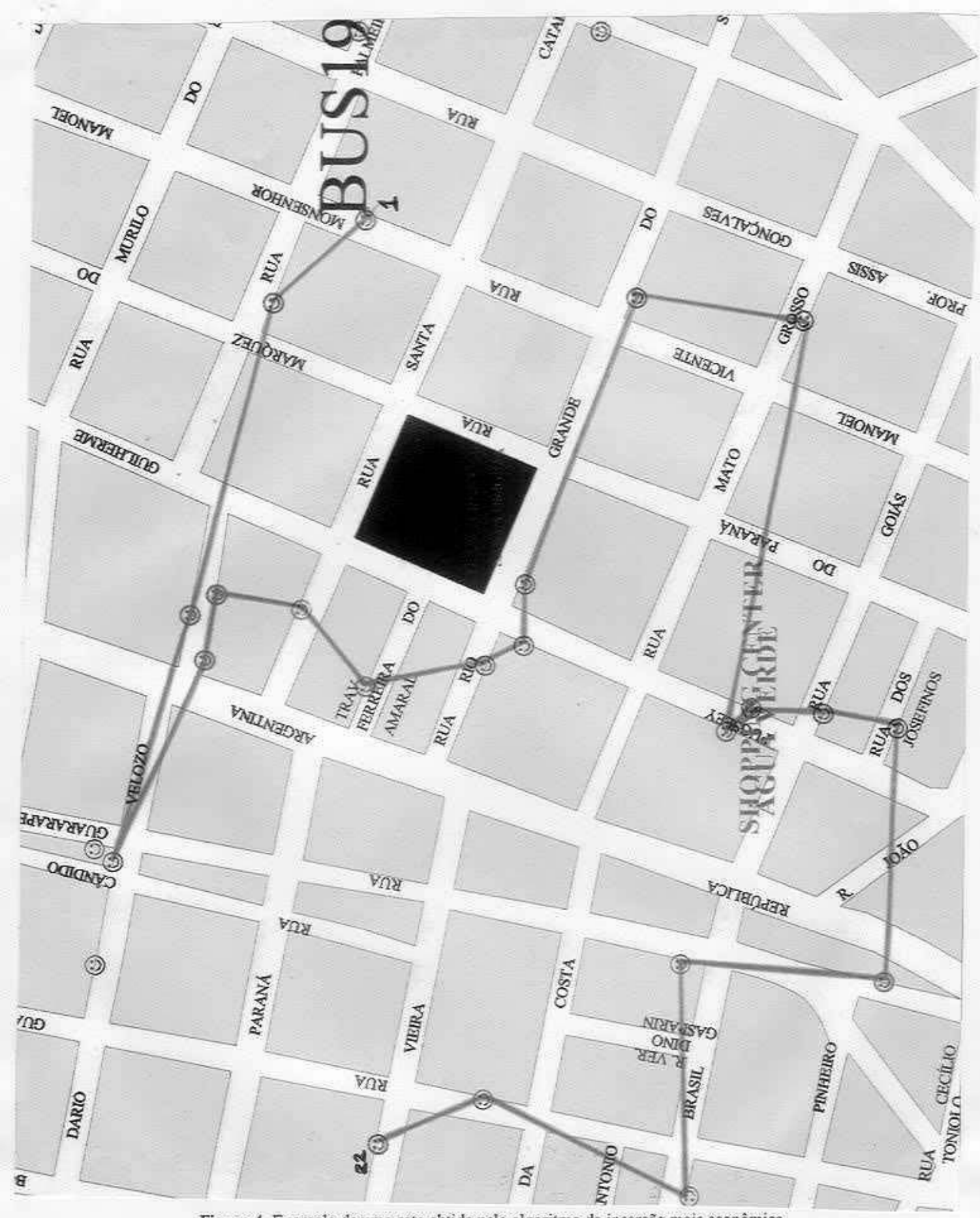

Figura 4. Exemplo de uma rota obtida pelo algoritmo da inserção mais econômica, a nivel de quadras (ônibus 19 - demanda $=30$ e capacidade $=32$ lugares). 


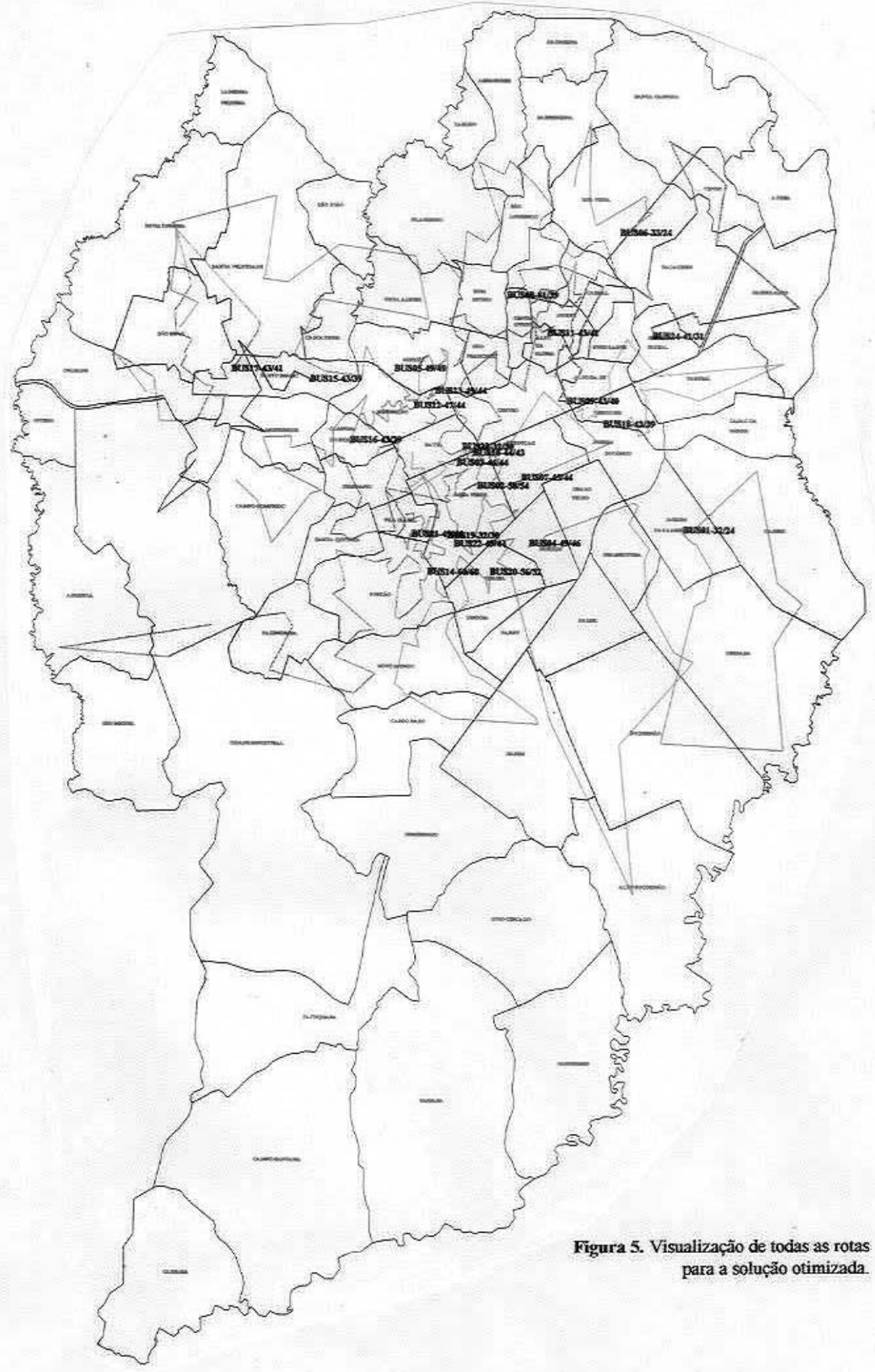

